\title{
The Schwartz kernel theorem for the tempered distributions on the Heisenberg group
}

\author{
Yasuyuki OKA \\ (Received July 2, 2013; Revised December 11, 2013)
}

\begin{abstract}
The aim of this paper is to give the Schwartz kernel theorem for the space of the tempered distributions on the Heisenberg group.

Key words: Tempered distributions on the Heisenberg group, Heat equation, Schwartz kernel theorem, BIBO stability.
\end{abstract}

\section{Introduction}

The Heisenberg group is the non-commutative Lie group. The harmonic analysis on the Heisenberg group is studied by several mathematicians ([2], $[3],[4],[6],[7],[8],[9],[12],[16],[17],[18]$ and so on).

The aim of this article is to give the Schwartz kernel theorem for the tempered distributions on the Heisenberg group by means of the heat kernel method on the Heisenberg group. The heat kernel method, introduced by T. Matsuzawa in [13], is the method to characterize the generalized functions on the Euclidean space by the initial value of the solutions of the heat equation. Especially, in [14], T. Matsuzawa showed the heat kernel method for the tempered distributions on the Euclidean space. On the other hand, in [11], J. Kim and M. W. Wong showed the heat kernel method for the tempered distributions on the Heisenberg group. We apply the heat kernel method for the tempered distributions on the Heisenberg group to the proof of the Schwartz kernel theorem for the tempered distributions on the Heisenberg group.

As an application of the Schwartz kernel theorem for the tempered distributions, R. Ashino, T. Mandai and A. Morimoto constructed a continuous linear time shift invariant system and considered BIBO (Bounded-Input Bounded-Output) stability of the continuous linear time shift invariant system on the Euclidean space in [1]. Under this idea, we consider BIBO stability of a continuous linear system constructed by the tempered kernel distribution on the Heisenberg group. 


\section{The Heisenberg group $\mathbb{H}^{d}$}

First of all, we fix some notations. We use a multi-index $\alpha \in \mathbb{Z}_{+}^{d}$, namely, $\alpha=\left(\alpha_{1}, \ldots, \alpha_{d}\right)$, where $\alpha_{i} \in \mathbb{Z}$ and $\alpha_{i} \geq 0$. So, for $x \in \mathbb{R}^{d}$, $x^{\alpha}=x_{1}^{\alpha_{1}} \cdots x_{d}^{\alpha_{d}}$ and $\partial_{x}^{\alpha}=\partial_{x_{1}}^{\alpha_{1}} \cdots \partial_{x_{d}}^{\alpha_{d}}$, where $\partial_{x_{j}}^{\alpha_{j}}=\left(\partial / \partial x_{j}\right)^{\alpha_{j}}$. Moreover $\Delta=\sum_{j=1}^{d}\left(\partial^{2} / \partial x_{j}^{2}\right)$.

Let $(x, y, t)$ and $\left(x^{\prime}, y^{\prime}, t^{\prime}\right) \in \mathbb{R}^{d} \times \mathbb{R}^{d} \times \mathbb{R}=\mathbb{R}^{2 d+1}$. Then we define the group law of $\mathbb{R}^{2 d+1}$ by

$$
(x, y, t)\left(x^{\prime}, y^{\prime}, t^{\prime}\right)=\left(x+x^{\prime}, y+y^{\prime}, t+t^{\prime}+2\left(x^{\prime} \cdot y-x \cdot y^{\prime}\right)\right),
$$

where $x \cdot y=\sum_{j=1}^{d} x_{j} y_{j}$. The group $\mathbb{R}^{2 d+1}$ with respect to the group law defined by (2.1) is called the Heisenberg group and denoted by $\mathbb{H}^{d}$. Its identity element is $(0,0,0)$ and the inverse of the element $(x, y, t)$ is $(x, y, t)^{-1}=(-x,-y,-t)$. The Heisenberg group $\mathbb{H}^{d}$ is a locally compact Hausdorff group and its Haar measure is the Lebesgue measure $d x d y d t$.

The left-invariant vector fields on the Heisenberg group $\mathbb{H}^{d}$ as $\mathbb{R}^{2 d+1}$ are represented by

$$
X_{j}=\frac{\partial}{\partial x_{j}}+2 y_{j} \frac{\partial}{\partial t}, \quad X_{d+j}=\frac{\partial}{\partial y_{j}}-2 x_{j} \frac{\partial}{\partial t} \text { and } X_{2 d+1}=\frac{\partial}{\partial t}
$$

for $j=1,2, \ldots, d$ and these make a basis for the Lie algebra of $\mathbb{H}^{d}$.

The sub-Laplacian $\Delta_{\mathbb{H}^{d}}$ on $\mathbb{H}^{d}$ is defined by

$$
\Delta_{\mathbb{H}^{d}}=\sum_{j=1}^{2 d} X_{j}^{2}
$$

We consider the heat operator

$$
\frac{\partial}{\partial s}-\Delta_{\mathbb{H}^{d}}
$$

on $\mathbb{H}^{d} \times(0, \infty)$.

Let $\lambda>0$. Then we define the dilations $\delta_{\lambda}$ by

$$
\delta_{\lambda}(x, y, t)=\left(\lambda x, \lambda y, \lambda^{2} t\right)
$$


for $(x, y, t) \in \mathbb{H}^{d}$.

The homogeneous dimension $Q$ of $\mathbb{H}^{d}$ is given by

$$
Q=2 d+2
$$

Moreover, a function $u$ from $\mathbb{H}^{d}$ to $\mathbb{C}$ is called the Heisenberghomogeneous of degree $k \in \mathbb{Z}$ if $u \circ \delta_{\lambda}=\lambda^{k} u$ for $\lambda>0$. Especially the Heisenberg-homogeneous of degree of the distance function $d$ defined by

$$
d(x, y, t)=\left(\left(x^{2}+y^{2}\right)^{2}+t^{2}\right)^{1 / 4}
$$

for $(x, y, t) \in \mathbb{H}^{d}$ is one, that is,

$$
d\left(\lambda x, \lambda y, \lambda^{2} t\right)=\lambda d(x, y, t)
$$

The distance between two points $(x, y, t)$ and $\left(x^{\prime}, y^{\prime}, t^{\prime}\right)$ in $\mathbb{H}^{d}$ is given by

$$
d\left(\left(x^{\prime}, y^{\prime}, t^{\prime}\right)^{-1}(x, y, t)\right)
$$

Let $f$ and $g$ be suitable functions on $\mathbb{H}^{d}$. Then we define the convolution $f * g$ of $f$ with $g$ as follows:

$$
(f * g)(x, y, t)=\int_{\mathbb{H}^{d}} f\left(x^{\prime}, y^{\prime}, t^{\prime}\right) g\left(\left(x^{\prime}, y^{\prime}, t^{\prime}\right)^{-1}(x, y, t)\right) d x^{\prime} d y^{\prime} d t^{\prime}
$$

for $(x, y, t),\left(x^{\prime}, y^{\prime}, t^{\prime}\right) \in \mathbb{H}^{d}$. The convolution on $\mathbb{H}^{d}$ is non-commutative, in general.

\section{The space $\mathcal{S}\left(\mathbb{H}^{d}\right)$ and its dual space $\mathcal{S}^{\prime}\left(\mathbb{H}^{d}\right)$}

Let $\alpha \in \mathbb{Z}_{+}^{2 d}$. Then the functions $\left(X_{\alpha} \varphi\right)(x, y, t)$ are defined by

$$
\left(X_{\alpha} \varphi\right)(x, y, t)=\left(X_{1}^{\alpha_{1}} X_{2}^{\alpha_{2}} \ldots X_{2 d}^{\alpha_{2 d}} \varphi\right)(x, y, t)
$$

for a function $\varphi \in C^{\infty}\left(\mathbb{H}^{d}\right)$.

We define the Schwartz class $\mathcal{S}\left(\mathbb{H}^{d}\right)$ on the Heisenberg group as follows:

Definition 1 For any $\varphi \in C^{\infty}\left(\mathbb{H}^{d}\right)$, we say $\varphi \in \mathcal{S}\left(\mathbb{H}^{d}\right)$ if the function $\varphi$ satisfies the following condition: For any $N \in \mathbb{Z}_{+}$, we have 


$$
\|\varphi\|_{N}=\sup _{(x, y, t) \in \mathbb{H}^{d}}(1+d(x, y, t))^{N} \sum_{|\alpha| \leq N}\left|X_{\alpha} \varphi(x, y, t)\right|<\infty .
$$

It is clear from the definition that the space $\mathcal{S}\left(\mathbb{H}^{d}\right)$ is topologically isomorphic of the space $\mathcal{S}\left(\mathbb{R}^{2 d+1}\right)$. Moreover, it is known that the Schwartz class $\mathcal{S}\left(\mathbb{H}^{d}\right)$ is a Fréchet space in [3].

Definition 2 We denote by $\mathcal{S}^{\prime}\left(\mathbb{H}^{d}\right)$ the dual space of the space $\mathcal{S}\left(\mathbb{H}^{d}\right)$ and call it the space of the tempered distributions on the Heisenberg group. Thus, $u \in \mathcal{S}^{\prime}\left(\mathbb{H}^{d}\right)$ if and only if $u$ is a linear functional from $\mathcal{S}\left(\mathbb{H}^{d}\right)$ to $\mathbb{C}$ and satisfies the following condition: There exist $N \in \mathbb{Z}_{+}$and a positive constant $C$ such that

$$
|\langle u, \varphi\rangle| \leq C \|_{N}
$$

for any $\varphi \in \mathcal{S}\left(\mathbb{H}^{d}\right)$.

By the definition, we can see that the space $\mathcal{S}^{\prime}\left(\mathbb{H}^{d}\right)$ is topologically isomorphic of the space $\mathcal{S}^{\prime}\left(\mathbb{R}^{2 d+1}\right)$.

Let $\check{f}(x, y, t)=f\left((x, y, t)^{-1}\right)$ for $(x, y, t) \in \mathbb{H}^{d}$. Then we define the convolution $u * \varphi$ of $u \in \mathcal{S}^{\prime}\left(\mathbb{H}^{d}\right)$ with $\varphi \in \mathcal{S}\left(\mathbb{H}^{d}\right)$ as follows:

$$
\langle u * \varphi, \psi\rangle=\langle u, \psi * \check{\varphi}\rangle
$$

for any $\psi \in \mathcal{S}\left(\mathbb{H}^{d}\right)$.

\section{The heat kernel method for the space $\mathcal{S}^{\prime}\left(\mathbb{H}^{d}\right)$}

In [6] and [9], we can find the explicit form of the heat kernel (the fundamental solutions) $P_{s}(x, y, t)$ of the heat operator

$$
\frac{\partial}{\partial s}-\Delta_{\mathbb{H}^{d}}
$$

on $\mathbb{H}^{d}$ as follows:

$$
P_{s}(x, y, t)=\left\{\begin{array}{lr}
(4 \pi s)^{-(d+1)} \int_{-\infty}^{\infty}\left(\frac{2 \tau}{\sinh 2 \tau}\right)^{d} e^{(i \tau t / 2 s)-\left(2\left(|x|^{2}+|y|^{2}\right) \tau / 4 s \tanh 2 \tau\right)} d \tau \\
0, & s>0 \\
& s \leq 0 .
\end{array}\right.
$$


The following properties of the heat kernel $P_{s}(x, y, t)$ hold:

Proposition 1 ([5]) Let $P_{s}$ be the heat kernel associated to the subLaplacian $\Delta_{\mathbb{H}^{d}}$. Then the following properties hold:

(i) $P_{s}(x, y, t) \geq 0$,

(ii) $\int_{\mathbb{H}^{d}} P_{s}(x, y, t) d x d y d t=1$,

(iii) $P_{s}(x, y, t)=P_{s}\left((x, y, t)^{-1}\right)$,

(iv) $\left(\frac{\partial}{\partial s}-\Delta_{\mathbb{H}^{d}}\right) P_{s}(x, y, t)=0$,

(v) $\lim _{s \rightarrow+0} P_{s}=\delta$ in $\mathcal{S}^{\prime}\left(\mathbb{H}^{d}\right)$,

(vi) $P_{r^{2} s}\left(r x, r y, r^{2} t\right)=r^{-Q} P_{s}(x, y, t), r>0$.

Moreover the heat kernel $P_{s}(x, y, t)$ has the following estimate:

Proposition 2 ([10]) Let $P_{s}(x, y, t)$ be the heat kernel associated to the sub-Laplacian $\Delta_{\mathbb{H}^{d}}$. Then for any $\alpha \in \mathbb{Z}_{+}^{2 d}$ and $m \in \mathbb{Z}_{+}$, there exist positive constant $a$ and $C_{m, \alpha}$ such that

$$
\left|\frac{\partial^{m}}{\partial s^{m}} X_{\alpha} P_{s}(x, y, t)\right| \leq C_{m, \alpha} s^{-m-|\alpha| / 2-Q / 2} e^{-a d(x, y, t)^{2} / s} .
$$

Proposition 3 The heat kernel $P_{s}(x, y, t)$ is in the space $\mathcal{S}\left(\mathbb{H}^{d}\right)$ for $s>0$. Moreover for any $\varphi \in \mathcal{S}\left(\mathbb{H}^{d}\right)$, the following property holds:

$$
\varphi * P_{s} \rightarrow \varphi \in \mathcal{S}\left(\mathbb{H}^{d}\right)
$$

as $s$ converges to +0 .

Proof. Let $g=(x, y, t), g^{\prime}=\left(x^{\prime}, y^{\prime}, t^{\prime}\right) \in \mathbb{H}^{d}$. Then it is enough to show that we have for any $N_{1} \in \mathbb{Z}_{+}, \alpha \in \mathbb{Z}_{+}^{d}$,

$$
\lim _{s \rightarrow+0} \sup _{g \in \mathbb{H}^{d}} d(g)^{N_{1}}\left|X_{\alpha}\left(\varphi * P_{s}\right)(g)-X_{\alpha} \varphi(g)\right|=0
$$

for $\varphi \in \mathcal{S}\left(\mathbb{H}^{d}\right)$.

Since 


$$
d(g)^{N_{1}} \leq 2^{N_{1}}\left(d\left(g^{\prime-1} g\right)^{N_{1}}+d\left(g^{\prime}\right)^{N_{1}}\right)
$$

by Proposition 1 , we have

$$
\begin{aligned}
& d(g)^{N_{1}}\left|X_{\alpha}\left(\varphi * P_{s}\right)(g)-X_{\alpha} \varphi(g)\right| \\
& =\left|d(g)^{N_{1}} \int_{\mathbb{H}^{d}} X_{\alpha} \varphi\left(g^{\prime}\right) P_{s}\left(g^{\prime-1} g\right) d g^{\prime}-X_{\alpha} \varphi(g)\right| \\
& \leq 2^{N_{1}} \int_{\mathbb{H}^{d}} d\left(g^{\prime-1} g\right)^{N_{1}} P_{s}\left(g^{\prime-1} g\right)\left|X_{\alpha} \varphi\left(g^{\prime}\right)-X_{\alpha} \varphi(g)\right| d g^{\prime} \\
& \quad+2^{N_{1}} \int_{\mathbb{H}^{d}} d\left(g^{\prime}\right)^{N_{1}} P_{s}\left(g^{\prime-1} g\right)\left|X_{\alpha} \varphi\left(g^{\prime}\right)-X_{\alpha} \varphi(g)\right| d g^{\prime} \\
& =(\mathrm{I})+(\mathrm{II}) .
\end{aligned}
$$

By Proposition (5.4) in [5], for any $\varepsilon>0$, there exists $\delta>0$ such that if $d\left(g^{\prime-1} g\right) \leq \delta$, then we have

$$
\sup _{g, g^{\prime} \in \mathbb{H}^{d}}\left|X_{\alpha} \varphi\left(g^{\prime}\right)-X_{\alpha} \varphi(g)\right| \leq \varepsilon
$$

Moreover since $\varphi \in \mathcal{S}\left(\mathbb{H}^{d}\right)$, there exists a positive constant $M_{\alpha}$ such that

$$
\sup _{g \in \mathbb{H}^{d}}\left|X_{\alpha} \varphi(g)\right| \leq M_{\alpha}
$$

Now we consider the integral in (I) in the case $\left(\mathrm{I}_{\mathrm{a}}\right) ; d\left(g^{\prime-1} g\right)>\delta$ or in the case $\left(\mathrm{I}_{\mathrm{b}}\right) ; d\left(g^{\prime-1} g\right) \leq \delta$. In the case $\left(\mathrm{I}_{\mathrm{a}}\right)$, by Proposition 2 , we have

$$
\begin{aligned}
& 2^{N_{1}} d\left(g^{-1} g\right)^{N_{1}} P_{s}\left(g^{\prime-1} g\right) \\
& \quad \leq C_{N_{1}} d\left(g^{\prime-1} g\right)^{N_{1}} s^{-(d+1)} e^{\left.-\left(a d\left(g^{\prime-1} g\right)^{2}\right) / s\right)} \\
& \quad \leq C_{N_{1}} d\left(g^{\prime-1} g\right)^{N_{1}} e^{-\left(a d\left(g^{\prime-1} g\right)^{2}\right) / 2 s} s^{-(d+1)} e^{-\left(a d\left(g^{\prime-1} g\right)^{2}\right) / 2 s} \\
& \quad \leq C_{N_{1}}^{\prime} s^{N_{1} / 2} s^{-(d+1)} e^{-\left(a d\left(g^{\prime-1} g\right)^{2}\right) / 2 s} .
\end{aligned}
$$

Hence by $(2.2),(4.1)$ and 


$$
\int_{\mathbb{H}^{d}} e^{-a d(G)^{2}} d G<\infty
$$

we obtain

$$
\begin{aligned}
2^{N_{1}} & \int_{d\left(g^{\prime-1} g\right)>\delta} d\left(g^{-1} g\right)^{N_{1}} P_{s}\left(g^{\prime-1} g\right)\left|X_{\alpha} \varphi\left(g^{\prime}\right)-X_{\alpha} \varphi(g)\right| d g^{\prime} \\
& \leq C_{N_{1}, \alpha}^{\prime \prime} s^{N_{1} / 2} s^{-(d+1)} \int_{d\left(g^{\prime-1} g\right)>\delta} e^{-\left(a d\left(g^{\prime-1} g\right)^{2}\right) / 2 s} d g^{\prime} \\
& \leq C_{N_{1}, \alpha}^{\prime \prime} s^{N_{1} / 2} s^{-(d+1)} \int_{\mathbb{H}^{d}} e^{-\left(a d\left(g^{\prime-1} g\right)^{2}\right) / 2 s} d g^{\prime} \\
& =C_{N_{1}, \alpha}^{\prime \prime} s^{N_{1} / 2} \int_{\mathbb{H}^{d}} e^{-a d(G)^{2}} d G \\
& =C_{N_{1}, \alpha}^{\prime \prime \prime} s^{N_{1} / 2} \\
& \rightarrow 0
\end{aligned}
$$

as $s$ converges to +0 . On the other hand, in the case $\left(\mathrm{I}_{\mathrm{b}}\right)$, for any $\varepsilon>0$, we have

$$
\begin{aligned}
& 2^{N_{1}} \int_{d\left(g^{\prime-1} g\right) \leq \delta} d\left(g^{\prime-1} g\right)^{N_{1}} P_{s}\left(g^{\prime-1} g\right)\left|X_{\alpha} \varphi\left(g^{\prime}\right)-X_{\alpha} \varphi(g)\right| d g^{\prime} \\
& \quad \leq \tilde{C}_{N_{1}, \alpha} \varepsilon \delta \int_{\mathbb{H}^{d}} P_{s}\left(g^{\prime-1} g\right) d g^{\prime} \\
& \quad=\tilde{C}_{N_{1}, \alpha} \varepsilon \delta .
\end{aligned}
$$

In the case (II), we can obtain the same result by a similar argument in the case (I). Therefore we have

$$
\varphi * P_{s} \rightarrow \varphi
$$

in $\mathcal{S}\left(\mathbb{H}^{d}\right)$ as $s$ converges to +0 .

J. Kim and M. W. Wong obtained the following characterization of the space $\mathcal{S}^{\prime}\left(\mathbb{H}^{d}\right)$. This characterization is called "the heat kernel method":

Theorem 1 ([11]) For $u \in \mathcal{S}^{\prime}\left(\mathbb{H}^{d}\right)$, we put 


$$
U_{s}(x, y, t)=\left(u * P_{s}\right)(x, y, t)
$$

for $(x, y, t) \in \mathbb{H}^{d}$ and $s>0$. Then the function $U_{s}(x, y, t)$ satisfies the following four conditions:

( i ) $U_{s}(x, y, t) \in C^{\infty}\left(\mathbb{H}^{d} \times(0, \infty)\right)$,

(ii) $\left(\frac{\partial}{\partial s}-\Delta_{\mathbb{H}^{d}}\right) U_{s}(x, y, t)=0,(x, y, t) \in \mathbb{H}^{d}$ and $s>0$,

(iii) for any $\varphi \in \mathcal{S}\left(\mathbb{H}^{d}\right)$,

$$
\langle u, \varphi\rangle=\lim _{s \rightarrow+0} \int_{\mathbb{H}^{d}} U_{s}(x, y, t) \varphi(x, y, t) d x d y d t
$$

and

(iv) there exist $\mu, \nu>0$ and a constant $C>0$ such that

$$
\left|U_{s}(x, y, t)\right| \leq C s^{-\mu}(1+d(x, y, t))^{\nu}, 0<s<1,
$$

for $(x, y, t) \in \mathbb{H}^{d}$.

Conversely every $U_{s}(x, y, t) \in C^{\infty}\left(\mathbb{H}^{d} \times(0, \infty)\right)$ satisfying the conditions (ii) and (iv) can be expressed in the form

$$
U_{s}(x, y, t)=\left(u * P_{s}\right)(x, y, t)
$$

with the unique element $u \in \mathcal{S}^{\prime}\left(\mathbb{H}^{d}\right)$.

Example 1 Let $u$ be the Dirac's delta function $\delta \in \mathcal{S}^{\prime}\left(\mathbb{H}^{d}\right)$. Then we have

$$
U_{s}(x, y, t)=\left(\delta * P_{s}\right)(x, y, t)=P_{s}(x, y, t)
$$

Hence by Proposition 2, there exist positive constants $a$ and $C$ such that

$$
\left|U_{s}(x, y, t)\right|=\left|P_{s}(x, y, t)\right| \leq C s^{-d-1} e^{-\left(a d(x, y, t)^{2}\right) / s} .
$$

\section{Schwartz kernel theorem for the space $\mathcal{S}^{\prime}\left(\mathbb{H}^{d}\right)$}

As an application of the characterization of the space $\mathcal{S}^{\prime}\left(\mathbb{H}^{d}\right)$, we show the Schwartz kernel theorem for the space $\mathcal{S}^{\prime}\left(\mathbb{H}^{d}\right)$ as follows: 
Theorem 2 Let $k$ be a continuous linear operator from $\mathcal{S}\left(\mathbb{H}^{d_{2}}\right)$ to $\mathcal{S}^{\prime}\left(\mathbb{H}^{d_{1}}\right)$. Then there exists $T$ in $\mathcal{S}^{\prime}\left(\mathbb{H}^{d_{1}} \times \mathbb{H}^{d_{2}}\right)$ such that

$$
\langle k \psi, \varphi\rangle=\langle T, \varphi \otimes \psi\rangle
$$

where $\varphi$ is in $\mathcal{S}\left(\mathbb{H}^{d_{1}}\right)$ and $\psi$ is in $\mathcal{S}\left(\mathbb{H}^{d_{2}}\right)$.

Proof. Since $k$ is continuous, the bilinear form $\mathbb{B}$ on $\mathcal{S}\left(\mathbb{H}^{d_{1}}\right) \times \mathcal{S}\left(\mathbb{H}^{d_{2}}\right)$,

$$
\mathbb{B}(\varphi, \psi)=\langle k \psi, \varphi\rangle, \quad \varphi \in \mathcal{S}\left(\mathbb{H}^{d_{1}}\right), \quad \psi \in \mathcal{S}\left(\mathbb{H}^{d_{2}}\right)
$$

is separately continuous. Since $\mathcal{S}\left(\mathbb{H}^{d_{1}}\right)$ and $\mathcal{S}\left(\mathbb{H}^{d_{2}}\right)$ are the Fréchet space respectively, $\mathbb{B}$ is continuous. Hence we can see that there exist a positive constant $C$ and $N_{1}, N_{2} \in \mathbb{Z}_{+}$such that

$$
|\langle k \psi, \varphi\rangle| \leq C\|\varphi\|_{N_{1}}\|\psi\|_{N_{2}} .
$$

We define $R_{s}\left(\left(x_{1}, y_{1}, t_{1}\right),\left(x_{2}, y_{2}, t_{2}\right)\right)$ by

$$
\begin{aligned}
& R_{s}\left(\left(x_{1}, y_{1}, t_{1}\right),\left(x_{2}, y_{2}, t_{2}\right)\right) \\
& \quad=\left\langle k P_{s}\left(\left(x_{2}, y_{2}, t_{2}\right)^{-1}(\cdot, \cdot, \cdot)\right), P_{s}\left(\left(x_{1}, y_{1}, t_{1}\right)^{-1}(\cdot, \cdot, \cdot)\right)\right\rangle
\end{aligned}
$$

for $\left(\left(x_{1}, y_{1}, t_{1}\right),\left(x_{2}, y_{2}, t_{2}\right)\right) \in \mathbb{H}^{d_{1}} \times \mathbb{H}^{d_{2}}$ and $s>0$.

Now we show $R_{s}$ converges in $\mathcal{S}^{\prime}\left(\mathbb{H}^{d_{1}} \times \mathbb{H}^{d_{2}}\right)$ as $s \rightarrow+0$. By $(\sharp)$, there exist a positive constant $C$ and $M, N_{1}, N_{2} \in \mathbb{Z}_{+}$such that

$\left|R_{s}\left(\left(x_{1}, y_{1}, t_{1}\right),\left(x_{2}, y_{2}, t_{2}\right)\right)\right| \leq C s^{-M}\left(1+d\left(x_{1}, y_{1}, t_{1}\right)\right)^{N_{1}}\left(1+d\left(x_{2}, y_{2}, t_{2}\right)\right)^{N_{2}}$, for $\left(x_{1}, y_{1}, t_{1}\right) \in \mathbb{H}^{d_{1}},\left(x_{2}, y_{2}, t_{2}\right) \in \mathbb{H}^{d_{2}}$ and $0<s<1$.

Moreover we obtain

$$
\left(\frac{\partial}{\partial s}-\Delta_{\mathbb{H}^{d_{1}+d_{2}}}\right) R_{s}\left(\left(x_{1}, y_{1}, t_{1}\right),\left(x_{2}, y_{2}, t_{2}\right)\right)=0
$$

for $\left(x_{1}, y_{1}, t_{1}\right) \in \mathbb{H}^{d_{1}},\left(x_{2}, y_{2}, t_{2}\right) \in \mathbb{H}^{d_{2}}$ and $0<s<1$.

Therefore, by Theorem 1 , there exists $R_{0} \in \mathcal{S}^{\prime}\left(\mathbb{H}^{d_{1}} \times \mathbb{H}^{d_{2}}\right)$ such that

$$
R_{0}=\lim _{s \rightarrow+0} R_{s}
$$


in $\mathcal{S}^{\prime}\left(\mathbb{H}^{d_{1}} \times \mathbb{H}^{d_{2}}\right)$.

Since the Riemann sum of an integral converges in $\mathcal{S}\left(\mathbb{H}^{d_{j}}\right), j=1,2$, by Proposition 1, we have

$$
\begin{aligned}
\left\langle R_{s}, \varphi \otimes \psi\right\rangle= & \iint_{\mathbb{H}^{d_{1} \times \mathbb{H}^{d_{2}}}} R_{s}\left(\left(x_{1}, y_{1}, t_{1}\right),\left(x_{2}, y_{2}, t_{2}\right)\right) \varphi\left(x_{1}, y_{1}, t_{1}\right) \psi\left(x_{2}, y_{2}, t_{2}\right) \\
& \times d x_{1} d y_{1} d t_{1} d x_{2} d y_{2} d t_{2} \\
& =\left\langle k \int_{\mathbb{H}^{d_{2}}} P_{s}\left(\left(x_{2}, y_{2}, t_{2}\right)^{-1}(\cdot, \cdot, \cdot)\right) \psi\left(x_{2}, y_{2}, t_{2}\right) d x_{2} d y_{2} d t_{2},\right. \\
& \left.\int_{\mathbb{H}^{d_{1}}} P_{s}\left(\left(x_{1}, y_{1}, t_{1}\right)^{-1}(\cdot, \cdot, \cdot)\right) \varphi\left(x_{1}, y_{1}, t_{1}\right) d x_{1} d y_{1} d t_{1}\right\rangle \\
= & \left\langle k\left[\psi * P_{s}\right], \varphi * P_{s}\right\rangle
\end{aligned}
$$

for $\varphi \in \mathcal{S}\left(\mathbb{H}^{d_{1}}\right)$ and $\psi \in \mathcal{S}\left(\mathbb{H}^{d_{2}}\right)$.

Therefore by Proposition 3, we obtain

$$
\left\langle R_{0}, \varphi \otimes \psi\right\rangle=\langle k \psi, \varphi\rangle
$$

as $s \rightarrow+0$.

\section{The translation invariant operator}

Let $g=(x, y, t) \in \mathbb{H}^{d}$. Then we define the translation operator $T_{\beta}$ by

$$
\left[T_{\beta} f\right](g)=f\left(\beta^{-1} g\right)
$$

for $\beta \in \mathbb{H}^{d}$. A continuous linear map $k: \mathcal{S}\left(\mathbb{H}^{d}\right) \rightarrow \mathcal{S}^{\prime}\left(\mathbb{H}^{d}\right)$ is said to be translation invariant if

$$
T_{\beta} k[\psi]=k\left[T_{\beta} \psi\right]
$$

for any $\psi \in \mathcal{S}\left(\mathbb{H}^{d}\right)$.

By Theorem 2, for any continuous linear system (map) $k$ from $\mathcal{S}\left(\mathbb{H}^{d}\right)$ to $\mathcal{S}^{\prime}\left(\mathbb{H}^{d}\right)$, there exists the tempered kernel distribution $K \in \mathcal{S}^{\prime}\left(\mathbb{H}^{d} \times \mathbb{H}^{d}\right)$ such that

$$
\langle k \psi, \varphi\rangle=\langle K, \varphi \otimes \psi\rangle
$$


for $\varphi, \psi \in \mathcal{S}\left(\mathbb{H}^{d}\right)$.

Now we have the following Proposition 4:

Proposition 4 Let $k: \mathcal{S}\left(\mathbb{H}^{d}\right) \rightarrow \mathcal{S}^{\prime}\left(\mathbb{H}^{d}\right)$ be a continuous linear system and $K\left(g_{1}, g_{2}\right)$ be its tempered kernel distribution on $\mathbb{H}^{d} \times \mathbb{H}^{d}$. The system $k$ is translation invariant if and only if there exists $h \in \mathcal{S}^{\prime}\left(\mathbb{H}^{d}\right)$ such that

$$
K\left(g_{1}, g_{2}\right)=h\left(g_{2}^{-1} g_{1}\right)
$$

that is,

$$
k[\psi]=\psi * h
$$

for any $\psi \in \mathcal{S}\left(\mathbb{H}^{d}\right)$.

Proof. Let us prove the converse first. If

$$
K\left(g_{1}, g_{2}\right)=h\left(g_{2}^{-1} g_{1}\right)
$$

for some $h \in \mathcal{S}^{\prime}\left(\mathbb{H}^{d}\right)$, then it is clear to see that

$$
k[\psi]=\psi * h
$$

is a translation invariant.

Let us assume that $k$ is translation invariant. Then for any $\psi \in \mathcal{S}\left(\mathbb{H}^{d}\right)$,

$$
\begin{aligned}
\left\langle K\left(\beta^{-1} g_{1}, g_{2}\right), \psi\left(g_{2}\right)\right\rangle & =\left\langle K\left(g_{1}, g_{2}\right), \psi\left(\beta^{-1} g_{2}\right)\right\rangle \\
& =\left\langle K\left(g_{1}, \beta g_{2}\right), \psi\left(g_{2}\right)\right\rangle
\end{aligned}
$$

for $\beta \in \mathbb{H}^{d}$. Hence we have

$$
K\left(\beta^{-1} g_{1}, g_{2}\right)=K\left(g_{1}, \beta g_{2}\right)
$$

in $\mathcal{S}^{\prime}\left(\mathbb{H}^{d}\right)$, that is,

$$
K\left(g_{1}, g_{2}\right)=K\left(\beta g_{1}, \beta g_{2}\right)
$$

So we obtain

$$
\langle K, \phi\rangle=\left\langle K, \phi\left(\beta^{-1} g_{1}, \beta^{-1} g_{2}\right)\right\rangle
$$


for any $\phi \in \mathcal{S}^{\prime}\left(\mathbb{H}^{d} \times \mathbb{H}^{d}\right)$. Therefore this completes the proof from the following Lemma 1.

Lemma 1 If $K \in \mathcal{S}^{\prime}\left(\mathbb{H}^{d} \times \mathbb{H}^{d}\right)$ satisfies (6.1), then there exists $h \in \mathcal{S}^{\prime}\left(\mathbb{H}^{d}\right)$ so that

$$
K\left(g_{1}, g_{2}\right)=h\left(g_{2}^{-1} g_{1}\right)
$$

Proof. Let $\psi \in \mathcal{S}\left(\mathbb{H}^{d}\right)$ so that

$$
\int_{\mathbb{H}^{d}} \psi(\tilde{g}) d \tilde{g}=1
$$

Assume that $h$ satisfies

$$
K\left(g_{1}, g_{2}\right)=h\left(g_{2}^{-1} g_{1}\right)
$$

Since we have

$$
h\left(\xi_{1}\right)=K\left(\xi_{2} \xi_{1}, \xi_{2}\right)
$$

we can express

$$
\langle h, \phi\rangle=\left\langle K\left(\xi_{2} \xi_{1}, \xi_{2}\right), \phi\left(\xi_{1}\right) \psi\left(\xi_{2}\right)\right\rangle, \quad \phi \in \mathcal{S}\left(\mathbb{H}^{d}\right) .
$$

Next we will show the existence of $h$. We define $h \in \mathcal{S}^{\prime}\left(\mathbb{H}^{d}\right)$ by

$$
\left\langle h\left(g_{1}\right), \phi\left(g_{1}\right)\right\rangle:=\left\langle K\left(\xi_{2} \xi_{1}, \xi_{2}\right), \phi\left(\xi_{1}\right) \psi\left(\xi_{2}\right)\right\rangle, \quad \phi \in \mathcal{S}\left(\mathbb{H}^{d}\right) .
$$

By (6.4), we have for $f \in \mathcal{S}\left(\mathbb{H}^{d}\right)$,

$$
\begin{aligned}
\left\langle h\left(g_{2}^{-1} g_{1}\right), f\left(g_{2}\right) \phi\left(g_{1}\right)\right\rangle & =\left\langle h\left(g_{1}\right), f\left(g_{2}\right) \phi\left(g_{2} g_{1}\right)\right\rangle \\
& =\left\langle K\left(\xi_{2} \xi_{1}, \xi_{2}\right), f\left(g_{2}\right) \phi\left(g_{2} \xi_{1}\right) \psi\left(\xi_{2}\right)\right\rangle .
\end{aligned}
$$

By the coordinate change $\left(\xi_{1}, \xi_{2}, g_{2}\right) \rightarrow\left(\xi_{2}^{-1} \xi_{1}, \xi_{2}, g_{2} \xi_{2}\right)$, we obtain

$$
(6.5)=\left\langle K\left(\xi_{1}, \xi_{2}\right), f\left(g_{2} \xi_{2}\right) \phi\left(g_{2} \xi_{1}\right) \psi\left(\xi_{2}\right)\right\rangle .
$$

Thus, by (6.2), we have 


$$
(6.6)=\left\langle K\left(\xi_{1}, \xi_{2}\right), f\left(\xi_{2}\right) \phi\left(\xi_{1}\right) \psi\left(g_{2}^{-1} \xi_{2}\right)\right\rangle
$$

So, by (6.3), we obtain

$$
(6.7)=\left\langle K\left(\xi_{1}, \xi_{2}\right), f\left(\xi_{2}\right) \phi\left(\xi_{1}\right)\right\rangle \text {. }
$$

Hence we have

$$
K\left(g_{1}, g_{2}\right)=h\left(g_{2}^{-1} g_{1}\right)
$$

\section{BIBO stability}

The BIBO (Bounded-Input Bounded-Output) stability of the continuous linear system is defined as follows:

Definition 3 The continuous linear system $k$ is said to be BIBO stable if there exists constant $C>0$ such that

$$
\sup _{g \in \mathbb{H}^{d}}|k[\psi](g)| \leq C \sup _{g \in \mathbb{H}^{d}}|\psi(g)|
$$

for any $\psi \in \mathcal{S}\left(\mathbb{H}^{d}\right)$.

Theorem 3 Let $k[\psi]=\psi * h$ for $h \in \mathcal{S}^{\prime}\left(\mathbb{H}^{d}\right)$ and $\psi \in \mathcal{S}\left(\mathbb{H}^{d}\right)$. Then $k$ is BIBO stable if and only if $\Lambda \in \mathcal{M}\left(\mathbb{H}^{d}\right)$, where $\Lambda \in \mathcal{S}^{\prime}\left(\mathbb{H}^{d}\right)$ is the kernel of $k$ and $\mathcal{M}\left(\mathbb{H}^{d}\right)$ denotes the space of bounded Radon measures on $\mathbb{H}^{d}$.

Proof. Since it is clear that the sufficient condition holds, it is enough to show the necessary. If $k$ is BIBO stable, then there exists a constant $C>0$ such that

$$
|k[\psi](\mathbb{E})| \leq C \sup _{g \in \mathbb{H}^{d}}|\psi(g)|
$$

for $\psi \in \mathcal{S}\left(\mathbb{H}^{d}\right)$, where $\mathbb{E}=(0,0,0)$. This implies that $\psi \longmapsto k[\psi](\mathbb{E})$ is a bounded Radon measure $\mu$ by Riesz's representation theorem (see [15]). Therefore we have

$$
k[\psi](\mathbb{E})=\langle\mu, \psi\rangle=\int_{\mathbb{H}^{d}} \psi\left(g_{1}\right) d \mu\left(g_{1}\right) .
$$


Since the map $k$ is translation invariant, we can see that for any $g \in \mathbb{H}^{d}$,

$$
k[\psi](g)=\langle\mu, \psi\rangle=\int_{\mathbb{H}^{d}} \psi\left(g_{1}\right) d \mu\left(g_{1}^{-1} g\right) .
$$

Example 2 Let $h=\delta \in \mathcal{S}^{\prime}\left(\mathbb{H}^{d}\right)$. Then we have

$$
k[\psi](g)=(\psi * \delta)(g)=\int_{\mathbb{H}^{d}} \psi\left(g^{\prime}\right) \delta\left(g^{\prime-1} g\right) d g^{\prime}=\psi(g), \quad \psi \in \mathcal{S}\left(\mathbb{H}^{d}\right) .
$$

Thus we can see that the kernel of $k$ can be expressed by the Dirac's measure and $k$ is BIBO stable.

Acknowledgement The author is grateful to Professor Kunio Yoshino for his reading of the manuscript and useful suggestions.

\section{References}

[1] Ashino R., Mandai T. and Morimoto A., System identification based on distribution theory and wavelet transform. Applicable Anal., 84(2) (2005), 165-195.

[ 2 ] Calin O., Chang D.-C. and Greiner P., Geometric Analysis on the Heisenberg Group and Its Generalizations, AMS/IP, (2007).

[ 3 ] Corwin L. and Greenleaf F. P., Representations of Nilpotent Lie Groups and Their Applications: Volume 1, Part 1, Basic Theory and Examples, Cambridge University Press, Cambridge, (1990).

[ 4 ] Folland G. B., Harmonic Analysis in Phase Space, Princeton University Press. Princeton, N.J., (1989).

[5] Folland G. B., Subelliptic estimates and function spaces on nilpotent Lie group. Ark. Mat. 13 (1975), 161-207.

[6] Gaveau B., Principe de moindre action, propagation de la chaleur, et estimées sous elliptiques sur certains groupes nilpotents. Acta Math. 139 (1977), 95-153.

[ 7 ] Geller D., Fourier analysis on the Heisenberg group. I. Schwartz space. J. Funct. Anal. 36 (1980), 205-254.

[ 8 ] Howe R., On the role of the Heisenberg group in harmonic analysis. Bull. Amer. Math. Soc. 3 (1980), 821-843.

[9] Hulanicki A., The distribution of energy in the Brownian motion in the Gaussian field and analytic hypoellipticity of certain subelliptic operators on the Heisenberg group. Studia Math. 56 (1976), 165-173. 
[10] Jerison D. S. and Sánchez-Calle A., Estimates for the heat kernel for a sum of squares of vector fields. Indiana Univ. Math. J. 35 (1986), 835-854.

[11] Kim J. and Wong M. W., Positive definite temperature functions on the Heisenberg group. Appl. Anal. 85(8) (2006), 987-1000.

[12] Koranyi A., Geometric aspects of analysis on the Heisenberg group, in Topics in modern harmonic analysis, vol. II. Proc. Sem. Torino and Milano (1982), 209-258.

[13] Matsuzawa T., A calculus approach to the hyperfunctions I. Nagoya Math. J. 108 (1987), 53-66.

[14] Matsuzawa T., A calculus approach to the hyperfunctions III. Nagoya Math. J. 118 (1990), 133-153.

[15] Rudin W., Real and Complex Analysis, McGraw-Hill, Inc., New York, Tokyo, (1966).

[16] Stein E. M., Harmonic Analysis: Real-Variable Methods, Orthogonality, and Oscillatory Integrals, Princeton University Press, Princeton, N. J., (1993).

[17] Thangavelu S., An Introduction to the Uncertainty Principle: Hardy's Theorem on Lie Groups, Birkhäuser, Boston, (2004).

[18] Wong M. W., Weyl Transforms, Springer-Verlag, New York, (1998).

General Education

Kushiro National College of Technology

2-32-1 Otanoshike-Nishi, Kushiro-Shi, Hokkaido 084-0916, Japan

E-mail: oka@kushiro-ct.ac.jp 